\title{
The Influence of Cardiac Pacemaker Programming Modes on Exercise Capacity
}

\author{
BOGDAN CALOIAN ${ }^{1}$, ADELA VIVIANA SITAR-TAUT ${ }^{2}$, GABRIEL NICOLAE GUSETU ${ }^{1}$, \\ DANA POP $^{1}$ and DUMITRU TUDOR ZDRENGHEA ${ }^{1}$ \\ ${ }^{1}$ Cardiology-Rehabilitation Department, Clinical Rehabilitation Hospital, and \\ ${ }^{2}$ Internal Medicine Department, Medical Clinic IV, \\ Iuliu Hatieganu University of Medicine and Pharmacy, Cluj-Napoca, Romania
}

\begin{abstract}
Background/Aim: The cardiac pacing mode influences the atrioventricular synchronicity and the response of the heart rate to physical exercise. The aim of this study was to compare the influence of the most common pacemaker programming modes on exercise capacity. Patients and Methods: Fifty-two pacemaker-wearing patients were clinically evaluated and submitted to an exercise stress test. Results: Symptoms of heart failure were more frequently met in the single-chamber pacemaker group compared to the dual-chamber group. The parameters recorded during the exercise stress test were significantly better with the rate responsive function (RRF) activated. The effort time was higher by an average of 2.1 minutes and the exercise capacity was higher by 0.92 metabolic equivalents. Conclusion: Dual-chamber pacing is superior to singlechamber (ventricular) pacing and the activation of the RRF in single-chamber pacemakers has similar impact on exercise capacity as the preservation of atrioventricular synchronicity by dual-chamber pacemakers.
\end{abstract}

A pacemaker not only has the role of preventing the heart rate from dropping under a certain limit, but can also have an important influence on the exercise capacity (1), as the pacing mode influences atrioventricular synchronicity (single-chamber $v s$. dual-chamber pacemakers) $(2,3)$ and the

This article is freely accessible online.

Correspondence to: Dana Pop, Clinical Rehabilitation Hospital, Viilor str., No. 46-50, Cardiology Department, 2nd floor, room 222, Cluj-Napoca, Cluj, Romania. Tel: +40 264207021, Fax: +40 264453353, e-mail: pop67dana@gmail.com

Key Words: Cardiac pacemaker, pacemaker programming modes, single-chamber pacing, dual-chamber pacing, rate responsive function, exercise capacity. response of the heart rate to physical exercise (rate responsive function, mode switch function) $(4,5)$.

Most patients who have a pacemaker implanted for sick sinus syndrome or for atrioventricular blocks need to be enrolled in cardiac rehabilitation programs in order to improve their exercise capacity and quality of life. One of the most important components of cardiac rehabilitation is physical exercise, a programme that needs to be individualized for each patient by taking into account various factors such as age, gender, risk factors, comorbidities, and fitness level (6).

The first step in prescribing a physical training plan is the evaluation of the maximum exercise capacity of the patient through a standardized exercise stress test, noting the symptoms that forced the patient to stop the test, the maximum workload, the variations of the patient's blood pressure, heart rate, oxygen saturation, significant arrhythmias or ischemic changes recorded on the electrocardiographic (ECG) monitoring (7-9).

Besides the parameters mentioned above, the pacemakerwearing patient needs a more complex assessment during exercise because some particular events might occur: the heart rate might not adapt to exercise in patients with chronotropic incompetence, resulting in early left ventricular failure; atrioventricular conduction disturbances could worsen, causing symptoms such as dyspnoea, dizziness or even syncope; tachyarrhythmia or ischemic changes can be difficult to diagnose on an ECG recording during paced atrial or ventricular beats $(10,11)$.

In the present study, we focused on the exercise capacity of patients with cardiac pacemakers and on the benefits or the disadvantages brought by choosing a particular programming mode. Most of the studies that evaluated the exercise capacity of pacemaker-wearing patients enrolled a limited number of individuals and returned discordant results.

The purpose of our study was to compare the influence of the most common pacemaker programming modes on the exercise capacity of the patients. 


\section{Materials and Methods}

We conducted the study at the Cardiology Department of the Clinical Rehabilitation Hospital in Cluj-Napoca, Romania, including patients with a pacemaker implanted for more than 6 months and for whom we performed a cycloergometer exercise stress test following the adjusted Bruce protocol (12).

The anamnesis, complete physical examination, electrocardiography and echocardiography were performed before the exercise stress testing, during the same hospital admission. Symptoms and signs of heart failure were evaluated according to the 2016 European Society of Cardiology Guidelines for the diagnosis and treatment of acute and chronic heart failure (13).

Echocardiographic examinations were performed using either Philips Affiniti 70 Ultrasound Machine (Koninklijke Philips N.V., Amsterdam, Holland) or Esaote MyLab50 Ultrasound Machine (Esaote SpA, Genoa, Italy). The left atrial and left ventricular enddiastolic diameters were measured from parasternal long axis view and the ejection fraction was determined with Simpson's biplane formula (14).

Patients with comorbidities that could significantly impair exercise capacity (respiratory, neurological, musculoskeletal, vascular or significant structural heart diseases) were excluded from the study. Treatment with beta-blockers was stopped 24 hours before the test and resumed immediately after.

Pacemaker type and programming. The patients enrolled in the present study were wearing single-chamber pacemakers: Sensia SESR01 Implantable Pulse Generator (Medtronic, Minneapolis, MN, USA) or Sustain XL SR (St. Jude Medical, Saint Paul, MN, USA), or dual-chamber pacemakers: SEDR01 Sensia DR (Medtronic) or Sustain XL DR (St. Jude Medical). The implanted devices mentioned above were interrogated to determine the programming parameters using Medtronic Pacemaker and ICD Programmer model 2090 (Medtronic) and St. Jude Medical Merlin Patient Care System (St. Jude Medical). The pacemaker programming parameters were not modified prior to the test, all patients that were tested remained with the pacemaker settings selected by their personal cardiologist.

Statistical analysis. The data obtained were statistically analysed using IBM SPSS Statistics Software version 23 (International Business Machines Corporation, Armonk, North Castle, New York, NY, USA) and Microsoft Office 2013 Excel Data Analysis (Microsoft Corp., Redmond, WA, USA) module. The chi-square test was used for qualitative (categorical) variables, while mean, standard deviation and Student $t$-test were applied for quantitative variables. The normality of all variables distribution was studied with the Kolmogorov-Smirnov test. To calculate the correlation coefficient, for variables with normal distribution, the ANOVA test was applied and for variables without a normal distribution, non-parametrical Kruskal-Wallis test was used. Statistic significance was considered present when the $p$-value was below 0.05 .

Ethics. The present clinical study was approved by the local Ethics Committee (approval number 115/6.01.2017) and was performed in accordance with the ethical standards laid down in the 1964 Declaration of Helsinki and its later amendments. All patients included in the present study gave their informed consent.

\section{Results}

We included in our study 52 patients, of whom 31 were males $(59.6 \%)$. Eleven patients were in atrial fibrillation $(21.1 \%)$, all wearing a single-chamber (ventricular) pacemaker. The baseline characteristics of the patients included in our study are presented in Table I.

When we analysed the patients taking into account the pacemaker type, either dual-chamber or single-chamber pacemaker, we found that at admission, the symptoms of heart failure were more frequently met in the single-chamber pacemaker group $(64.5 \%)$ compared to the dual-chamber pacemaker group $(42.85 \%)$, reaching statistical significance $(p=0.013)$. In the dual-chamber pacemaker group, the rate responsive function (RRF) of the pacemaker was nonsignificantly more frequently activated $(57.1 \%$ vs. $38.7 \%$, respectively, $p=0.22$ ).

Although the left ventricular ejection fraction was higher and the left atrial and left ventricular diameters were lower in the dual-chamber pacemaker group, the difference was not statistically significant.

When we analysed the impact of the activation of RRF on symptoms of heart failure, echocardiography parameters and exercise capacity, we observed the following: the symptoms of heart failure were more frequently met when RRF was deactivated $(67.85 \%$ vs. $41.67 \%, p=0.008)$; the left ventricular ejection fraction was higher and left atrial and left ventricular diameters were lower in patients with the RRF activated, but the differences did not reach statistical significance. The parameters recorded during the exercise stress test were significantly better with RRF activated. The effort time was higher by an average of 2.1 minutes $(p=0.013)$, the maximum workload was higher by an average of $21 \mathrm{~W}(p=0.029)$ and the exercise capacity estimated in metabolic equivalents (METs) was higher by an average of $0.92(p=0.034)$ (Table II).

There were four types of patients submitted to an exercise stress test considering their pacemaker programming mode: single-chamber without RRF activated (19 patients), singlechamber with RRF activated (12 patients), dual-chamber without RRF activated (nine patients) and dual-chamber with RRF activated (12 patients).

Patients in the group with dual-chamber with RRF had the best exercise capacity during the exercise stress test (5.56 \pm 1.18 METs), while patients in the group with singlechamber without RRF had the worst exercise capacity (3.97 $\pm 1.50 \mathrm{METs})$ and patients in groups with single-chamber with RRF and dual-chamber without RRF had intermediate values (5.07 \pm 1.56 and 5.28 \pm 1.65 , respectively) (Figure 1).

Backward multiple regression that included age, gender, pacing mode, resting heart rate, end diastolic left ventricular diameter, and left atrial diameter as variables identified the pacing programming mode as an independent predictor for 
Table I. Baseline characteristics of patients with dual-chamber (DDD) and single-chamber pacemakers (VVI). Dual chamber pacemakers have dual pacing (right atrium and right ventricle), dual sensing (right atrium and right ventricle), and dual response type (triggering and inhibition). Singlechamber pacemakers are able to perform ventricular pacing, ventricular sensing and respond by inhibition. Both pacemaker types can have rate responsive function activated or not.

\begin{tabular}{lccc}
\hline Characteristic & DDD $(\mathrm{n}=21)$ & VVI $(\mathrm{n}=31)$ & $\mathrm{p}$-Value \\
\hline Age, years & $66.67 \pm 6.53$ & $71 \pm 10.62$ & 0.075 \\
Male gender, $\mathrm{n}(\%)$ & $15(71.42 \%)$ & $16(51.61 \%)$ & 0.019 \\
Atrial fibrillation, $\mathrm{n}(\%)$ & $0(0 \%)$ & $11(35.48 \%)$ & 0.001 \\
Heart rate at rest, bpm & $66.19 \pm 8.05$ & $67.97 \pm 10.20$ & 0.487 \\
Symptoms of heart failure, $\mathrm{n}(\%)$ & $9(42.85 \%)$ & $20(64.51 \%)$ & 0.013 \\
Ejection fraction, \% & $52.48 \pm 7.10$ & $50.90 \pm 9.56$ & 0.500 \\
LA diameter, mm & $42.24 \pm 5.56$ & $45.10 \pm 8.30$ & 0.143 \\
LV end-diastolic diameter, mm & $50.57 \pm 7.97$ & $51.77 \pm 7.41$ & 0.580 \\
Rate responsive activated, $\mathrm{n}(\%)$ & $12(57.14 \%)$ & $12(38.70 \%)$ & 0.022 \\
\hline
\end{tabular}

LA: Left atrium, LV: left ventricle.

Table II. Influence of rate responsive function activation on clinical, echocardiography and exercise testing parameters.

\begin{tabular}{lccc}
\hline & \multicolumn{2}{c}{ Rate responsive function } & \\
\cline { 2 - 3 } Characteristic & Activated $(\mathrm{n}=24)$ & Deactivated $(\mathrm{n}=28)$ & \\
\hline Symptoms of heart failure, $\mathrm{n}(\%)$ & $10(41.67 \%)$ & $19(67.85 \%)$ & 0.008 \\
Ejection fraction, $\%$ & $52.33 \pm 6.53$ & $50.86 \pm 10.14$ & 0.543 \\
LA diameter, mm & $41.83 \pm 5.28$ & $45.75 \pm 8.50$ & 0.056 \\
LV end-diastolic diameter, mm & $49.37 \pm 4.97$ & $52.93 \pm 9.04$ & 0.093 \\
Effort time, min & $8.63 \pm 3.02$ & $6.54 \pm 2.81$ & 0.013 \\
Maximum workload, W & $98.33 \pm 31.65$ & $77.18 \pm 35.68$ & 0.029 \\
METs & $5.31 \pm 1.38$ & $4.39 \pm 1.64$ & 0.034 \\
\hline
\end{tabular}

LA: Left atrium, LV: left ventricle, METs: metabolic equivalents.

exercise capacity estimated in METs in men $(\mathrm{r}=0.51$, $p=0.014)$, but not in women $(\mathrm{r}=0.08, p=0.76)$ (Figure 2$)$.

\section{Discussion}

The pacemaker programming mode has an important influence on a patient's exercise capacity. Our results show that even though the patients had similar left ventricular ejection fraction, and left atrial and left ventricular diameters, the patients in whom the RRF of the pacemaker was activated had superior functional capacity proven by their exercise test parameters.

The results of previous clinical studies conducted in this field are discordant. Provenier and Jordaens (1994) showed that activating the RRF significantly increased the walking distance during a 6-minute walking test in 16 patients wearing a single chamber pacemaker (15).

On the contrary, in a more recently published article (2010), Uebing et al. stated that the activation of the same pacemaker function did not improve functional capacity in nine patients evaluated based on exercise test results (16).

Better functional capacity was also observed in our study in patients with dual-chamber pacemakers in comparison to single-chamber (ventricular) pacemakers, an expected result taking into account the fact that dual-chamber pacemakers preserve the atrioventricular synchronicity which increases cardiac output by up to $25 \%(17,18)$. Chabernaud et al. compared single-chamber and dual-chamber pacing modes in 10 patients, observing a superior exercise capacity with dual-chamber pacing mode (19).

In our study, the best exercise capacity was observed in patients with dual-chamber pacemaker with RRF activated, followed by those with dual-chamber without RRF activated and by single-chamber with RRF activated, the worst exercise capacity being noted in those with single-chamber pacemaker without RRF activated. Maity et al. concluded, after developing a clinical study with 10 patients, that the activation of RRF had a more considerable effect on the 


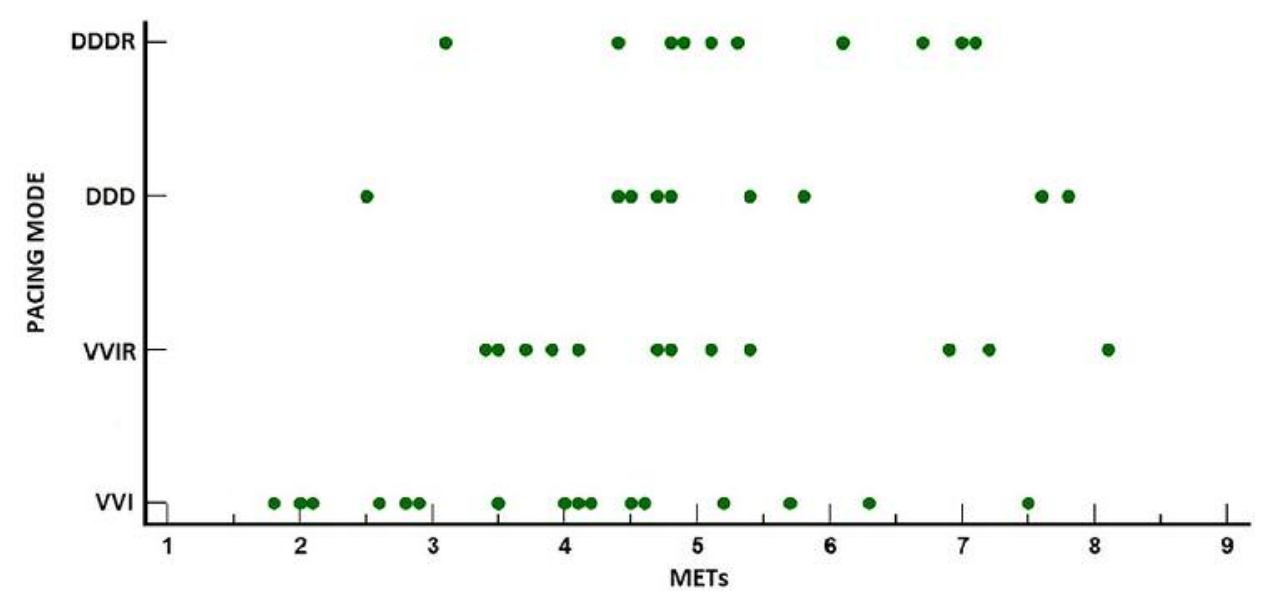

Figure 1. The influence of pacing programming modes on exercise capacity expressed in metabolic equivalents (METs). Patients wearing dualchamber pacemakers with rate responsive function (RRF) activated (DDDR) had the best exercise capacity, while those with single-chamber pacemakers without RRF activated (VVI) had the worst exercise capacity. There was no significant difference between dual-chamber pacing, RRF not activated (DDD) and single-chamber pacing, RRF activated (VVIR).

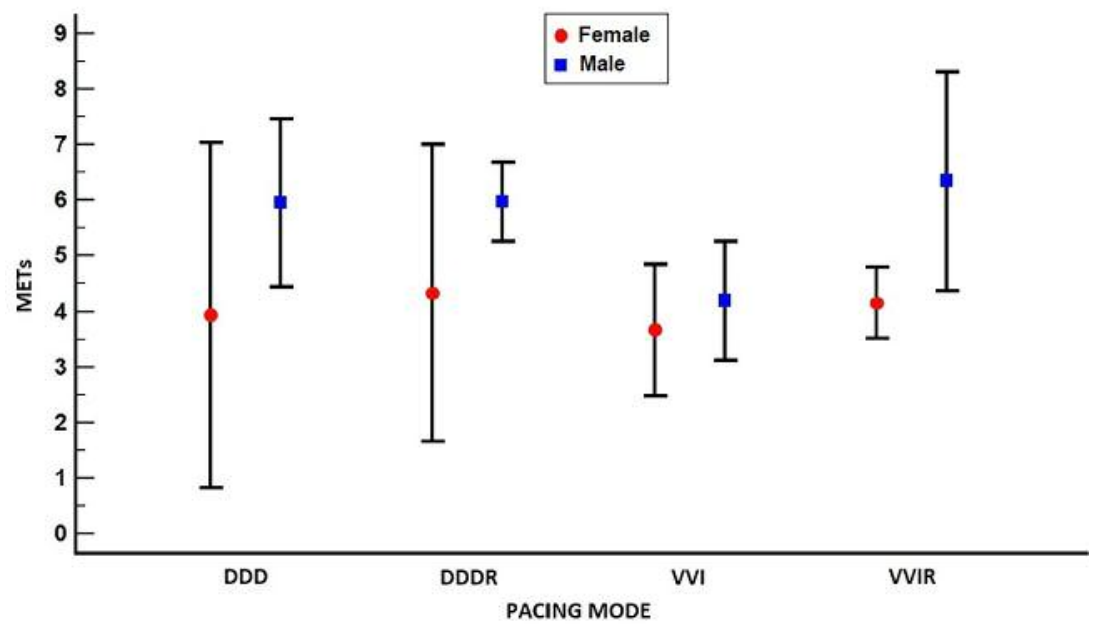

Figure 2. Gender differences in exercise capacity [mean metabolic equivalents (METS) and 95\% confidence interval] for the studied pacing modes. Pacing programming mode represents an independent predictor for exercise capacity in men, but not in women. VVI: Single-chamber pacemaker without rate responsive function (RRF) activated, VVIR: single-chamber pacemaker with RRF activated, DDD: dual-chamber pacemaker without RRF activated, DDDR: dual-chamber pacemaker with RRF activated.

exercise capacity than upgrading from single- to dualchamber pacing, although the upgrade also significantly improved the exercise test results (20).

The discordance between the results of previous clinical studies can be mainly explained by the small number of patients enrolled, and the fact that most of the patients wearing a pacemaker were unable to perform an exercise stress test because of the comorbidities associated with older age.
One of the most interesting and important observations of our research, also confirmed by other studies (21-24), is that patients with dual-chamber without RRF and those with single-chamber with RRF had similar exercise capacity. This means that the activation of a simple function of the pacemaker has the same benefits as preserving the contribution of the atrial contraction to cardiac output. In other words, a patient with permanent atrial fibrillation and 
a single-chamber pacemaker will be able to gain the exercise capacity of a patient wearing a dual-chamber pacemaker with preserved atrial contractions if the RRF is activated. On the other hand, a patient in sinus rhythm who receives a single-chamber ventricular pacemaker loses the benefits brought by atrial contraction and their exercise capacity descends to that of a patient with permanent atrial fibrillation. This observation is even more important if we take into account that RRF is not activated by default in all cardiac pacemaker types.

The activation of RRF proved to be beneficial irrespective of the presence of chronotropic incompetence, which was not taken into account, a fact that we consider a limitation of our study.

In women, the programming mode did not reach statistical significance as a predictor for exercise capacity, most probably because of the small number of female patients included in the study.

Taking into account that patients with single-chamber without RRF had the worst exercise capacity, the use of this programming mode should be limited only to cases in which comorbidities severely impair their physical activity. Otherwise, patients in atrial fibrillation should receive a single-chamber pacemaker with RRF activated and patients in sinus rhythm a similarly activated dual-chamber device.

In conclusion, the pacemaker programming mode significantly influences the exercise capacity. Dual-chamber pacing is superior to single-chamber (ventricular) pacing and the activation of the RRF in single-chamber pacemakers has a similar impact on exercise capacity as does preservation of atrioventricular synchronicity in dualchamber pacemakers. These observations should be considered by physicians when they program a pacemaker or prescribe physical rehabilitation.

\section{References}

1 Oliveira RM, da Silva KR, Kawauchi TS, Alves LB, Crevelari ES, Filho MM and Costa R: Functional capacity of patients with pacemaker due to isolated congenital atrioventricular block. Arq Bras Cardiol 104(1): 67-77, 2015.

2 Nielsen JC, Kristensen L, Andersen HR, Mortensen PT, Pedersen OL and Pedersen AK: A randomized comparison of atrial and dual-chamber pacing in 177 consecutive patients with sick sinus syndrome. J Am Coll Cardiol 42: 614-623, 2003.

3 Kinderman M, Hennen B, Jung J, Geisel J, Bohm M and Frohlig G: Biventricular versus conventional right ventricular stimulation for patients with standard pacing indication and left ventricular dysfunction: The Homburg Biventricular Pacing Evaluation (HOBIPACE). J Am Coll Cardiol 47: 1927-1937, 2006.

4 Palmisano P, Aspromonte V, Ammendola E, Delléra G, Ziacchi M, Guerra F, Aquilani S, Maglia G, Del Giorno G, Giubertoni A, Boriani G, Capucci A, Pietro Rici R and Accogli M: Effect of fixed-rate $v s$. rate-responsive pacing on exercise capacity in patients with permanent, refractory atrial fibrillation and left ventricular dysfunction treated with atrioventricular junction ablation and biventricular pacing (RESPONSIBLE): a prospective, multicentre, randomized, single-blind study. EP Europace 19(3): 414-420, 2017.

5 Passman RS, Weinberg KM, Freher M, Denes P, Schaechter A, Goldberger JJ and Kadish AH: Accuracy of mode switch algorithms for detection of atrial tachyarrhythmias. J Cardiovasc Electrophysiol 15: 773-777, 2004.

6 Greco EM, Guardini S and Citelli L: Cardiac rehabilitation in patients with rate responsive pacemakers. Pacing Clin Electrophysiol 21: 568-575, 1998.

7 Calvagna GM and Patane S: Cardiac rehabilitation in pacing patient complications: an increasing scenario requiring a collaborative vision of a multi-disciplinary treatment team. Int $\mathbf{J}$ Cardiol 178: 168-170, 2015.

8 Gostoli S, Roncuzzi R, Urbinati S and Rafanelli C: Clinical and subclinical distress, quality of life, and psychological well-being after cardiac rehabilitation. Appl Psychol Health Well Being 9(3): 349-369, 2017

9 Zdrenghea D, Ilea M, Pop D, Petrovai D, Bodisz G and Zdrenghea M: N-terminal pro B-type natriuretic peptide during exercise in patients with systolic and diastolic heart failure. Acta Endo 5(1): 19-25, 2009.

10 Zdrenghea D, Pop D and Gusetu G: The rehabilitation in cardiac resynchronisation therapy. In: Cardiac Rehabilitation Manual. Niebauer J. Springer International Publishing pp. 245-256, 2017.

11 Iliou MC, Blanchard JC, Lamar-Tanguy A, Cristofini P and Ledru F: Cardiac rehabilitation in patients with pacemakers and implantable cardioverter defibrillators. Monaldi Arch Chest Dis 86(1-2): 756, 2016.

12 Hill J and Timmis A: Exercise tolerance testing. BMJ 324(7345): 1084-1087, 2002.

13 Ponikowski P, Voors AA, Anker SD, Bueno H, Cleland JC, Coats AJS, Falk V, González-Juanatey JR, Harjola VP, Jankowska EA, Jessup M, Linde C, Nihoyannopoulos P, Parissis JT, Pieske B, Riley JP, Rosano GMC, Ruilope LM, Ruschitzka F, Rutten FH and van der Meer P: 2016 ESC Guidelines for the Diagnosis and Treatment of Acute and Chronic Heart Failure: The Task Force for the diagnosis and treatment of acute and chronic heart failure of the European Society of Cardiology (ESC) Developed with the special contribution of the Heart Failure Association (HFA) of the ESC. Eur Heart J 37(27): 2129-2200, 2016.

14 Schiller NB, Shah PN and Crawford M: Recommendations for quantitation of the left ventricle by two-dimensional echocardiography. J Am Soc Echocardiogr 2: 258-267, 1989.

15 Provenier F and Jordaens L: Evaluation of six minute walking test in patients with single chamber rate responsive pacemakers. Br Heart J 72(2): 192-196, 1994.

16 Uebing A, Diller GP, Li W, Maskell M, Dimopoulos K and Gatzoulis MA: Optimised rate-responsive pacing does not improve either right ventricular haemodynamics or exercise capacity in adults with a systemic right ventricle. Cardiol Young 20(5): 485-494, 2010.

17 Rodriguez Munoz DA, Moya Mur JL, Becker D, Fernandez Santos S, Lazaro Rivera C, Casas Rojo E, Navas Tejedor P, Delgado Montero A, Fernandez-Golfin C and Zamorano Gomez JL: Atrial systole contribution to cardiac output: more than just volume. Eur Heart J 34: 1122, 2013.

18 Klautz RJ, Baan J and Teitel DF: Contribution of synchronized atrial systole to left ventricular contraction in the newborn pig heart. Pediatr Res 43(3): 331-337, 1998. 
19 Chabernaud JM, Guéret P, Blanc P, Bavoux O and Bensaid J: Comparison of VVI and DDD cardiac stimulation during exercise test evaluated by respiratory gas exchange measurement. Study of patients with normal systolic function and complete atrioventricular block unchanged during exercise test. Arch Mal Coeur Vaiss 86(1): 69-74, 1993.

20 Maity AK, Ghosh SP, Dasbiswas A, Chatterjee SS, Chaudhury D and Das MK: Haemodynamic advantage with single-chamber rate responsive pacemakers over dual-chamber pacemakers during exercise in chronotropic incompetence. Indian Heart J: 44(4): 231-234, 1992.

21 Oldroyd KG, Rae AP, Carter R, Wingate C and Cobbe SM: Double blind crossover comparison of the effects of dual chamber pacing (DDD) and ventricular rate adaptive (VVIR) pacing on neuroendocrine variables, exercise performance, and symptoms in complete heart block. Br Heart J 65(4): 188-193, 1991.

22 Linde-Edelstam C, Hjemdahl P, Pehrsson SK, Aström H and Nordlander R: Is DDD pacing superior to VVIR? A study on cardiac sympathetic nerve activity and myocardial oxygen consumption at rest and during exercise. Pacing Clin Electrophysiol 15(4 Pt 1): 425-434, 1992.
23 Lau DH, Thiyagarajah A, Willems S, Rostock T, Linz D, Stiles MK, Kaye D, Kalman JM and Sanders P: Device therapy for rate control: pacing, resynchronisation and AV node ablation. Heart Lung Circ 26(9): 934-940, 2017.

24 Lau CP, Tai YT, Leung WH, Leung SK, Li JP, Wong CK, Lee IS, Yerich $\mathrm{C}$ and Erickson M: Rate adaptive cardiac pacing using right ventricular venous oxygen saturation: quantification of chronotropic behaviour during daily activities and maximal exercise. Pacing Clin Electrophysiol 17(12 Pt 1): 2236-2246, 1994.

Received December 2, 2017

Revised January 11, 2018

Accepted January 16, 2018 